\title{
Digital analysis of hormonal immunostaining in pituitary adenomas classified according to WHO 2017 criteria and correlation with preoperative laboratory findings
}

\author{
João Vitor Gerdulli Tamanini, ${ }^{1}$ Mateus Dal Fabbro, MD, ${ }^{2}$ Leandro Luiz Lopes de Freitas, MD, PhD, \\ José Vassallo, MD, PhD, ${ }^{1}$ Luciano de Souza Queiroz, MD, PhD, ${ }^{1}$ and Fabio Rogerio, MD, PhD ${ }^{1}$
}

Departments of ${ }^{1}$ Anatomical Pathology and ${ }^{2}$ Neurology, School of Medical Sciences, University of Campinas (UNICAMP), Campinas, São Paulo, Brazil

\begin{abstract}
OBJECTIVE The authors sought to evaluate clinical and laboratory data from pituitary adenoma (PA) patients with functioning PA (associated with acromegaly $[n=10]$ or Cushing disease $[n=10])$ or nonfunctioning PA (NFPA; $n=10)$ that were classified according to 2017 WHO criteria (based on the expression of the transcription factors pituitary-specific positive transcription factor 1 [Pit-1], a transcription factor member of the T-box family [Tpit], and steroidogenic factor 1 [SF-1]) and to assess the immunostaining results for growth hormone $(\mathrm{GH})$ and adrenocorticotropic hormone (ACTH) in the corresponding tumors.
\end{abstract}

METHODS Clinical and laboratory data were collected retrospectively. The percentage of tumoral cells positive for Pit-1, Tpit, or SF-1 was assessed and ImageJ software was used to evaluate immunopositivity in PAs with 2 different antibodies against $\mathrm{GH}$ (primary antibody 1 [AbGH-1] and primary antibody 2 [AbGH-2]) and 2 different antibodies against ACTH (primary antibody 1 [AbACTH-1] and primary antibody 2 [AbACTH-2]).

RESULTS Cells with positive Pit-1 staining were more frequently observed in lesions from patients with acromegaly (acromegaly group) than in lesions from patients with Cushing disease (Cushing group; $p<0.001$ ) and those from patients with NFPA (NFPA group; $p<0.001$ ). The percentage of Tpit-positive cells was higher in the Cushing group than in the acromegaly $(p<0.001)$ and NFPA ( $p<0.001)$ groups. No difference was detected regarding SF-1 frequency among all groups $(p=0.855)$. In acromegalic individuals, $\mathrm{GH}$ immunostaining levels varied depending on the antibody employed, and only one of the antibodies (AbGH-2) yielded higher values in comparison with the values for NFPA patients $(p<0.001)$. For all of the antibodies employed, no significant correlations were detected between $\mathrm{GH}$ tissue expression and the laboratory data (serum GH vs AbGH-1, $p=0.933$; serum $\mathrm{GH}$ vs AbGH-2, $p=0.853$; serum insulinlike growth factor-1 [IGF-1] vs AbGH-1, $p=0.407$; serum IGF-1 vs AbGH-2, $p=0.881$ ). In the Cushing group data, both antibodies showed similar ACTH tissue expression, which was higher than that obtained in the NFPA group $(p<0.001)$. There were no significant associations between ACTH immunohistochemical findings and ACTH serum levels (serum ACTH vs AbACTH-1, $p=0.651$; serum ACTH vs AbACTH-2, $p=0.987$ ). However, ACTH immunostaining evaluated with $\mathrm{AbACTH}-1$ showed a significant correlation with 24-hour urinary cortisol (24-hour cortisol vs AbACTH-1, $p=0.047$; 24hour cortisol vs AbACTH-2, $\mathrm{p}=0.071$ ).

CONCLUSIONS Immunostaining for Pit-1 and Tpit accurately identified lesions associated with acromegaly and Cushing disease, respectively. Conversely, SF-1 did not differentiate NFPA from lesions of the other two groups. Regarding hormonal tissue detection, results of the current investigation indicate that different antibodies may lead not only to divergent immunohistochemical results but also to lack of correlation with laboratory findings. Finally, PA classification based on transcription factor expression (Pit-1, Tpit, and SF-1), as proposed by the 2017 WHO classification of pituitary tumors, may avoid the limitations of PA classification based solely on digital immunohistochemical detection of hormones.

https://thejns.org/doi/abs/10.3171/2020.3.FOCUS2039

KEYWORDS acromegaly; Cushing disease; growth hormone; adrenocorticotropic hormone; Pit-1; Tpit; SF-1; immunohistochemistry

ABBREVIATIONS AbACTH-1 = primary antibody against ACTH (antibody 1); AbACTH-2 = primary antibody against ACTH (antibody 2); AbGH-1 = primary antibody against GH (antibody 1); AbGH-2 = primary antibody against GH (antibody 2); ACTH = adrenocorticotropic hormone; GH = growth hormone; HPF = high-power field; IGF-1 = insulin-like growth factor-1; NFPA = nonfunctioning pituitary adenoma; PA = pituitary adenoma; Pit-1 = pituitary-specific positive transcription factor 1 ; POMC = proopiomelanocortin; PRL = prolactin; SF-1 = steroidogenic factor 1 ; Tpit = a transcription factor member of the T-box family.

SUBMITTED January 30, 2020. ACCEPTED March 3, 2020.

INCLUDE WHEN CITING DOI: 10.3171/2020.3.FOCUS2039. 
$\mathrm{T}$ HE majority of sellar region tumors are pituitary adenomas (PAs), which may be clinically categorized as functioning or nonfunctioning PAs (NFPAs). ${ }^{26}$ NFPAs are not associated with endocrine syndromes secondary to hormonal hypersecretion..$^{2,9,11,27,33}$ On the other hand, functional PAs secrete hormones that lead to clinical manifestations and are classified as lesions that produce prolactin (PRL), growth hormone $(\mathrm{GH})$, adrenocorticotropic hormone (ACTH), or thyroid-stimulating hormone. . $, 9,11,24,26,27,33$ PAs that arise from gonadotrophin-secreting cells are uncommonly associated with endocrine manifestations and are included in the NFPA group. ${ }^{24,26,27}$

The 2017 WHO classification of pituitary tumors highlighted the importance of transcription factors in differentiating cellular lineage in adenomas..$^{20,26}$ In particular, pituitary-specific positive transcription factor 1 (Pit-1) is important in somatotroph differentiation, ${ }^{4}$ a transcription factor member of the T-box family (Tpit) identifies corticotroph cells, ${ }^{41}$ and steroidogenic factor 1 (SF-1) associates with gonadotroph lineage cells in PAs. ${ }^{3}$

Transsphenoidal resection is the initial treatment for patients with PAs, with the exception being PRL-secreting tumors, for which dopamine agonists are the main option. The PAs that are the most frequently treated surgically are NFPAs and those that secrete GH or ACTH. Specifically, NFPAs tend to manifest with hypopituitarism, visual deficits, and headache. GH-secreting lesions lead to acromegaly in adults, which is characterized by the growth of extremities and coarse facial features. ACTH-secreting PAs manifest with Cushing disease, presenting centripetal obesity, osteoporosis, hypertension, and violaceous striae. $^{27}$

The relation between immunohistochemical findings of hormone expression in PAs and corresponding clinical features and/or serum levels is debatable. In fact, other authors have reported a correlation between PRL in PAs and its blood concentration. The opposite finding, of no correlation, has also been described. ${ }^{5,12,13,37}$ In one series, patients presenting with the highest ratios of PRL immunopositivity also showed the highest PRL serum levels. ${ }^{35}$ A correlation between immunostaining for $\mathrm{GH}$ and its serum levels was reported by some research groups and not observed by others. ${ }^{13,30}$ In addition, a correlation between the expression of ACTH and the corresponding blood levels in ACTH-secreting PAs has not been identified. ${ }^{12} \mathrm{Fi}-$ nally, there have been reports on the lack of immunopositivity for $\mathrm{GH}$ in PAs from patients with acromegaly. ${ }^{19,38}$

Because of the inconsistencies regarding the associations between immunostaining findings and clinical laboratory data in patients with PA, we designed an original approach to assess hormone expression in surgical samples from such patients. First, each lesion was classified according to 2017 WHO criteria based on expression of the transcription factors Pit-1, Tpit, and SF-1. Then, 2 different antibodies against ACTH or GH were used in PA specimens from individuals with Cushing disease, acromegaly, or NFPAs, and the ImageJ public domain image processing tool was employed to evaluate immunopositivity. By using such accessible and objective approaches, we aimed to increase the chances of detecting relations among hormonal tissue expression, respective serum concentrations, and levels of functionally related molecules (serum insulinlike growth factor-1 [IGF-1] and 24-hour urinary cortisol).

\section{Methods}

\section{Study Design and Case Selection}

The present study was based on retrospective analyses of specimens obtained from patients who underwent endonasal transsphenoidal approach surgery and clinical follow-up for PAs at our institution between 2014 and 2017. For specimen selection, histological samples were reviewed. The most representative samples were identified and the paraffin blocks selected for immunohistochemical study.

Specimens from 30 PA patients $(n=30)$ were studied and divided into three groups based on the type of the lesion: PA associated with acromegaly $(\mathrm{n}=10)$, Cushing disease $(n=10)$, or NFPA $(n=10)$. Clinical, laboratory (GH, ACTH, and IGF-1 serum concentrations, as well as 24-hour urinary cortisol levels), and neuroimaging data were collected from medical records. Only surgical specimens stored in paraffin blocks from patients without restriction of sex and age and with a diagnosis of NFPA or PA associated with either acromegaly or Cushing disease were considered. For the immunohistochemical analyses performed for transcription factor detection, pituitary tissue specimens from autopsied individuals without a history of endocrine or neurological disease $(n=5)$ were used as controls. The study design was approved by the University of Campinas Ethics Committee (protocol 2.727.453).

\section{Histological and Immunostaining Analyses}

Histological sections ( $4 \mu \mathrm{m}$ ) were obtained from each block and stained with $\mathrm{H} \& \mathrm{E}$ for confirmation of lesion sampling. Additional serial sections were separately immunostained for GH, ACTH, Pit-1, Tpit, and SF-1. Briefly, the sections were incubated with 2 different primary antibodies against $\mathrm{GH}$ (antibody 1 [AbGH-1]: 1:200, clone $\mathrm{HGH}$, Spring Bioscience Corp., catalog no. E17470; and antibody 2 [AbGH-2]: 1:1, polyclonal, Bio SB, catalog no. BSB5568) or 2 different primary antibodies against ACTH (antibody 1 [AbACTH-1]: 1:400, clone SPM501, Spring Bioscience Corp., catalog no. E16451; and antibody 2 [AbACTH-2]: 1:1, clone BSB-25, Bio SB, catalog no. BSB5015). Sections of the same specimens were exposed to primary antibodies against Pit-1 (1:100, clone D-7, Santa Cruz Biotechnology, catalog no. sc-393943), Tpit (1:100, clone CL6251, Atlas Antibodies, catalog no. AMAb91409), or SF-1 (1:100, clone N1665, Invitrogen, catalog no. 434200). The secondary antibody and peroxidase were applied by using a detection system (AdvanceTMHRP, Dako, catalog no. K4068) for $30 \mathrm{~min}$ at $37^{\circ} \mathrm{C}$. The chromogenic substrate was 3,3-diaminobenzidine.

To classify the PA specimens according to the 2017 WHO criteria based on expression of transcription factors, 10 randomly selected high-power fields (HPFs; $\times 400$ ) from specimens with Pit-1, Tpit, and SF-1 immunostaining were examined per section. Positive cells were counted and described as percentages of the total of cells in the HPF. The average of the percentages of positive cells in 10 HPFs was calculated for each individual. 
To avoid interference with digital analysis of the immunohistochemical findings, no counterstaining was performed in GH- or ACTH-stained sections. Two different antibodies were employed for hormonal tissue detection to increase the chances of identifying correlations with the respective laboratory counterparts (serum GH, IGF-1, and $\mathrm{ACTH}$ as well as urinary cortisol). The antibodies for hormonal detection were chosen because they were the ones in current use in our institution. For transcription factor detection, which was used for tumor classification, 1 specific antibody was used for each factor.

Immunohistochemical evaluations of $\mathrm{GH}$ and $\mathrm{ACTH}$ were digitally performed as previously reported..$^{29}$ Briefly, staining was examined in 8-15 randomly selected HPFs $(\times 400)$ per section, considering the available amount of tissue per specimen. Photos corresponding to each HPF were captured by an optical system composed of an Axioplan 2 Zeiss microscope, an Olympus DP72 digital camera, and the Olympus cellSens Standard 1.14 software. Each captured field was processed through a public domain image processing tool (ImageJ software, version 1.50i) by enhancing binary contrast. Specifically, the original image was examined by an experienced neuropathologist who chose the adequate cutoff point (threshold) to preserve the original staining features (Fig. 1). The final results were values referred to by the software as Mean values, which correspond to an estimate of the stained area in a specific HPF. These values are associated with the stained area and obtained by applying an adequate threshold to each HPF. The Mean values are not indicated with a specific unit of measurement but are absolute numbers ranging from 0 (absolute absence of immunostaining) to 255 (the maximal value of the ImageJ software measurement scale, indicating that the entire field is stained). ${ }^{34}$

\section{Statistical Analysis}

Frequencies of the categorical variables (sex, histological type, and adenoma size [macro- or microadenomas]) were presented as percentage. For numerical variables (age at symptom onset, age at histological diagnosis, diameter of the lesion, and laboratory data), mean \pm standard deviation and median were used. For descriptions of the Mean values of each hormonal immunostaining and percentages of transcription factor-positive cells, means and standard deviations were employed. For comparison of transcription factor-positive cells or Mean values between the groups, the Kruskal-Wallis test followed by the Dunn post hoc test was used. The Wilcoxon signed-rank test was employed to compare Mean values obtained in the same group with different antibodies. Spearman's coefficient was used to verify correlations between numerical variables (laboratory data and Mean values). The level of significance adopted for the statistical tests was 5\% (p < 0.05 ). Statistical analysis was performed with the SAS 9.2 Software for Windows (2002-2008, SAS Institute Inc.).

\section{Results}

\section{Clinical, Imaging, Laboratory, and Histological Data}

Regarding patients with acromegaly, 90\% were female and $100 \%$ had macroadenomas (greater diameter

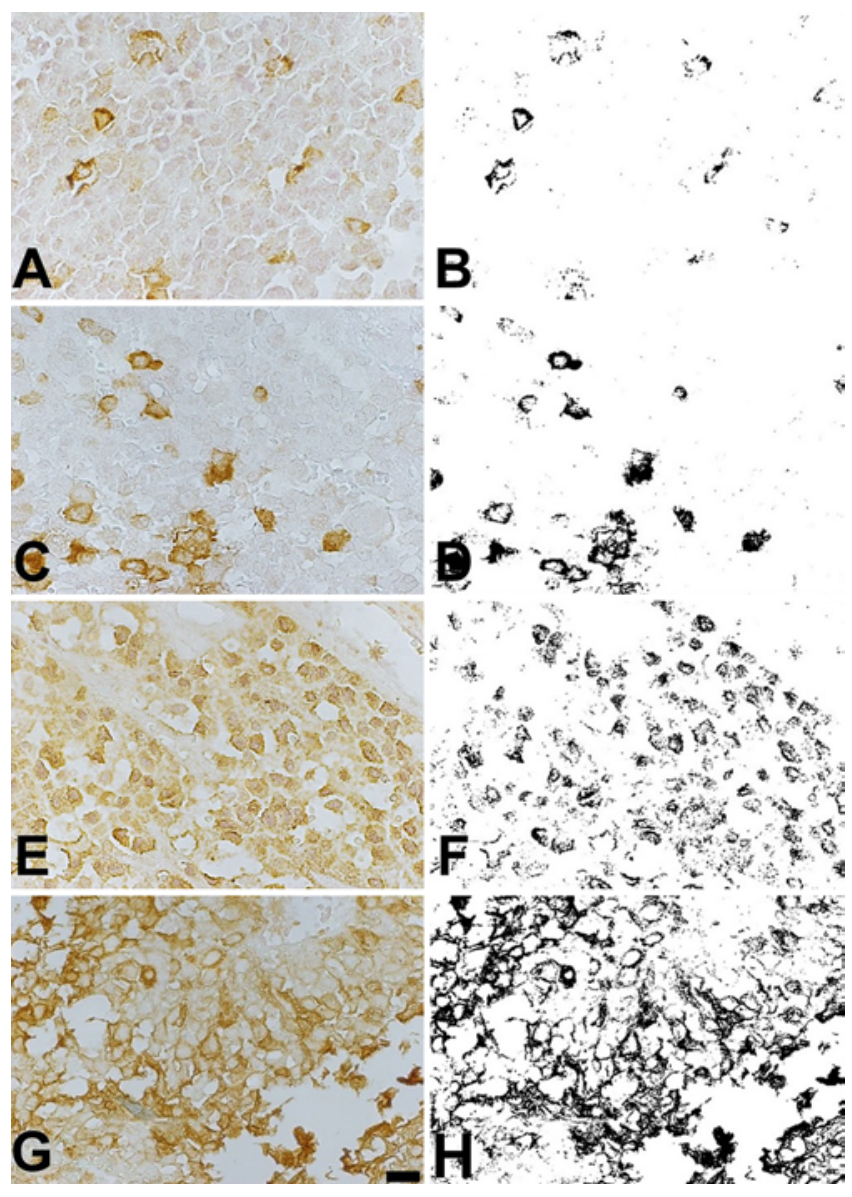

FIG. 1. Digital evaluation of immunostaining in PAs. Colored images of immunostained sections for $\mathrm{GH}(\mathbf{A}$ and $\mathbf{C})$ or $\mathrm{ACTH}(\mathbf{E}$ and $\mathbf{G})$ without counterstaining were digitally transformed into gray-scale images $(\mathrm{GH}$, $\mathbf{B}$ and $\mathbf{D} ; \mathrm{ACTH}, \mathbf{F}$ and $\mathbf{H}$ ). During such transformations, the software user applied a threshold so that the original immunohistochemical features were preserved. $A$ and $B, A b G H-1 ; C$ and $D, A b G H-2 ; E$ and $\mathrm{F}, \mathrm{AbACTH}-1 ; \mathrm{G}$ and $\mathrm{H}, \mathrm{AbACTH}-2$. Immunoperoxidase. Bar: $30 \mu \mathrm{m}$ $(\mathrm{A}-\mathrm{H})$.

$>10 \mathrm{~mm}$ ). The most frequent clinical manifestations were enlargement of extremities and coarse facial features. The ages at symptom onset and at histological diagnosis were $41.20 \pm 12.35$ years (median 38.00 years) and $45.90 \pm$ 12.39 years (median 41.50 years), respectively. On MRI, the greater diameter was $20.60 \pm 10.08 \mathrm{~mm}$ (median 19.00 $\mathrm{mm})$. GH and IGF-1 serum levels were $20.72 \pm 15.40 \mathrm{ng} /$ $\mathrm{ml}$ (median $18.71 \mathrm{ng} / \mathrm{ml}$ ) and $787.73 \pm 215.38 \mathrm{ng} / \mathrm{ml}$ (median $787.00 \mathrm{ng} / \mathrm{ml}$ ), respectively (Table 1). Concerning histological type, $50 \%$ of the PAs were chromophobic and $50 \%$ eosinophilic.

The majority of the individuals with Cushing disease were female $(80 \%)$ and had microadenomas (greater diameter $\leq 10 \mathrm{~mm}, 60 \%$ ). Obesity and muscle weakness were the main symptoms, which began at $27.50 \pm 7.95$ years of age (median 26.50 years). Histological diagnosis was performed at $30.30 \pm 9.95$ years (median 28.00 years). The greater diameter of the lesions on MRI was $12.70 \pm$ $14.10 \mathrm{~mm}$ (median $8.00 \mathrm{~mm}$ ). The 24-hour urinary cortisol levels were $487.84 \pm 217.40 \mu \mathrm{g} / 24 \mathrm{hrs}$ (median 505.70 
TABLE 1. Summary of laboratory and immunohistochemical data for each group of patient samples

\begin{tabular}{|c|c|c|c|}
\hline \multirow[b]{2}{*}{ PA Patient Data } & \multicolumn{3}{|c|}{ PA Patient Sample Group } \\
\hline & Acromegaly $(n=10)$ & Cushing Disease $(n=10)$ & NFPA $(n=10)$ \\
\hline \multicolumn{4}{|l|}{ Laboratory data } \\
\hline Serum $\mathrm{GH}, \mathrm{ng} / \mathrm{ml}$ & $20.72 \pm 15.40$ & - & - \\
\hline Serum IGF-1, ng/ml & $787.73 \pm 215.38$ & - & - \\
\hline Serum ACTH, pg/ml & - & $134.22 \pm 103.35$ & - \\
\hline Urinary cortisol, $\mu \mathrm{g} / 24 \mathrm{hrs}$ & - & $487.84 \pm 217.40$ & - \\
\hline \multicolumn{4}{|l|}{ Immunohistochemical data } \\
\hline \multicolumn{4}{|c|}{ Transcription factor, \% positive cells/HPF } \\
\hline Pit-1 & $68.74 \pm 24.09$ & $2.43 \pm 6.64$ & $4.35 \pm 13.60$ \\
\hline Tpit & 0 & $35.73 \pm 27.15$ & $0.24 \pm 0.75$ \\
\hline SF-1 & $38.77 \pm 39.08$ & $31.96 \pm 34.02$ & $24.16 \pm 24.72$ \\
\hline \multicolumn{4}{|c|}{ Hormone expression, Mean value } \\
\hline $\mathrm{AbGH}-1$ & $7.51 \pm 17.03$ & - & $8.72 \pm 10.46$ \\
\hline $\mathrm{AbGH}-2$ & $15.80 \pm 23.96$ & - & $0.01 \pm 0.02$ \\
\hline AbACTH-1 & - & $62.46 \pm 20.49$ & $0.36 \pm 0.99$ \\
\hline AbACTH-2 & - & $73.54 \pm 32.11$ & $0.02 \pm 0.01$ \\
\hline
\end{tabular}

Details of semiquantitative analyses of the immunohistochemical findings (percentage of positive cells and Mean value after digital assessment) for transcription factors (Pit-1, Tpit, and SF-1) and pituitary hormones (GH and ACTH) are given in the text. Mean values are presented \pm SD.

$\mu \mathrm{g} / 24 \mathrm{hrs})$, whereas ACTH serum levels were $134.22 \pm$ $103.35 \mathrm{pg} / \mathrm{ml}$ (median $112.30 \mathrm{pg} / \mathrm{ml}$; Table 1). On histological analysis, $50 \%$ of the lesions were basophilic, $30 \%$ chromophobic, and $20 \%$ eosinophilic.

Fifty percent of NFPA patients were female and all had macroadenomas. Symptoms were related to visual deficits, first noticed at age $51.00 \pm 14.08$ years (median 50.50 years). Histopathological diagnosis was made at age $54.30 \pm 15.36$ years (median 52.00 years). For MRI measurements, the greater diameter was $36.90 \pm 13.90$ $\mathrm{mm}$ (median $33.00 \mathrm{~mm}$ ). All of the lesions were chromophobic.

\section{Transcription Factors}

All transcription factors presented nuclear staining (Fig. 2). The average percentage of Pit-1-positive cells per HPF was $68.74 \% \pm 24.09 \%$ in patients with acromegaly, $2.43 \% \pm 6.64 \%$ for those with Cushing disease, and $4.35 \%$ $\pm 13.60 \%$ for patients with NFPAs (Table 1). PAs associated with acromegaly presented the highest frequency of Pit-1 positivity (acromegaly vs Cushing, $\mathrm{p}<0.001$; acromegaly vs NFPA, $\mathrm{p}<0.001$ ), whereas PAs associated with Cushing presented Pit-1 expression similar to that of NFPAs (p = 0.913; Fig. 3).

For Tpit, no positive cells were detected in acromegalic individuals. Cushing patients presented with $35.73 \%$ $\pm 27.15 \%$ and NFPA patients with $0.24 \% \pm 0.75 \%$ immunostained cells per HPF (Table 1). Cushing patients had the highest Tpit expression (Cushing vs acromegaly, $\mathrm{p}<$ 0.001 ; Cushing vs NFPA, $\mathrm{p}<0.001$ ), whereas Tpit expression was similar in the acromegaly and NFPA groups ( $\mathrm{p}$ $=0.311$; Fig. 3).

For SF-1, the values for acromegaly, Cushing, and NFPA were $38.77 \% \pm 39.08 \%, 31.96 \% \pm 34.02 \%$, and
$24.16 \% \pm 24.72 \%$ immunostained cells per HPF, respectively (Table 1). The values were statistically similar between all groups ( $\mathrm{p}=0.855$; Fig. 3 ).

\section{Tissue Expression of $\mathrm{GH}$ and $\mathrm{ACTH}$}

The staining pattern observed with both antibodies against GH was cytoplasmic and diffuse (Fig. 4). Tissue expression was assessed with ImageJ software. Mean values obtained from immunostaining with $\mathrm{AbGH}-1$ were $7.51 \pm 17.03$ and $8.72 \pm 10.46$ for patients presenting with acromegaly and NFPA, respectively. No significant difference was noted between those groups $(p=0.307)$. When using AbGH-2, hormonal tissue expressions (Mean values) in PA samples from individuals with acromegaly were $15.80 \pm 23.96$ and $0.01 \pm 0.02$ in NFPA, with a significant difference between such values $(\mathrm{p}<0.001$; Table 1$)$. Moreover, in acromegalic patients $\mathrm{GH}$ expression verified with AbGH-1 was significantly lower than that verified with AbGH-2 ( $p=0.020$; Fig. 4). Considering both acromegaly and NFPA groups together, there was no significant correlation between values obtained with AbGH-1 and those obtained with AbGH-2 ( $\mathrm{p}=0.626$; Fig. 5).

For the antibodies against ACTH, immunopositivity was noted in the cytoplasm with a diffuse distribution (Fig. 4). Tissue expression (Mean values) detected with AbACTH-1 was $62.46 \pm 20.49$ for patients with Cushing disease and $0.36 \pm 0.99$ for those with NFPA. The difference observed between those groups was significant ( $\mathrm{p}$ $<0.001$ ). The ACTH tissue expression (Mean values) observed with AbACTH-2 was $73.54 \pm 32.11$ in the Cushing group and $0.02 \pm 0.01$ in the NFPA group (Table 1). When comparing the two latter values, a significant difference was observed $(\mathrm{p}<0.001)$. Furthermore, ACTH expression was similar with both antibodies in patients with Cushing 


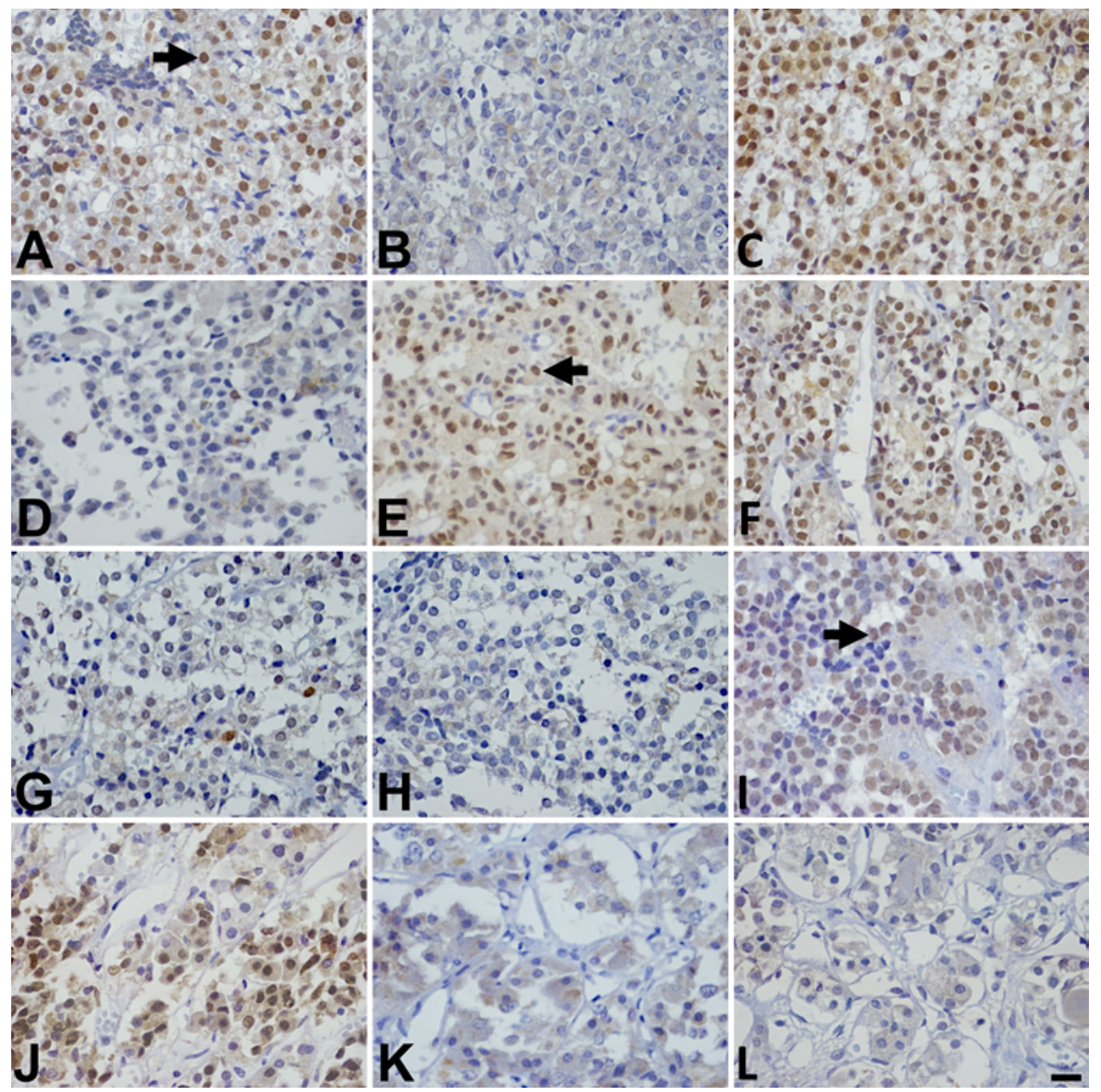

FIG. 2. Immunostaining patterns for Pit-1 (A, D, G, and J), Tpit (B, E, H, and K), and SF-1 (C, F, I, and L) in PAs from patients with acromegaly $(A-C)$, Cushing disease $(D-F)$, or NFPAs $(G-I)$, as well as in pituitary control samples $(J-L)$. Notice that the immunostaining pattern was nuclear for each antibody in PA (arrows) and control samples. Details on frequency of positive cells in each PA group are given in the text. Immunoperoxidase. Bar: $30 \mu \mathrm{m}(\mathrm{A}-\mathrm{L})$.

disease ( $\mathrm{p}=0.160$; Fig. 4). When both the Cushing and NFPA groups were considered together, correlations were observed between the values obtained with both antibodies ( $<<0.0001$; Fig. 6).

\section{Correlation Between Tissue Expression of GH or ACTH and Laboratory Data}

In acromegalic patients, GH tissue expression showed no correlation with hormone serum levels, irrespective of the antibody used (AbGH-1, p = 0.933; AbGH-2, p = 0.853). Additionally, IGF-1 levels did not correlate with GH lesion expression after both antibodies were tested (AbGH-1, p = 0.407; AbGH-2, p = 0.881; Fig. 5).

Individuals with Cushing disease had ACTH serum levels that did not correlate with tissue expression of the hormone detected by any antibody (AbACTH-1, $\mathrm{p}=0.651$; AbACTH-2, $p=0.987$ ). Finally, 24-hour urinary cortisol levels showed correlations with ACTH expression in PA when tested with AbACTH-1 $(\mathrm{p}=0.046)$ but not when tested with AbACTH-2 ( $p=0.071$; Fig. 6).

\section{Discussion}

We analyzed the tissue expression of GH and ACTH as well as their correlations with laboratory data from PAs classified according to the 2017 WHO criteria and obtained from patients with acromegaly, Cushing disease, or NFPAs. Clinical, neuroimaging, and laboratory findings from our patients were similar to those reported in previous studies. ${ }^{1,2,5,9,11,13,14,24,26,30,32,33,36}$ Additionally, Pit-1, Tpit, and SF-1 immunostaining patterns were nuclear, whereas $\mathrm{GH}$ and $\mathrm{ACTH}$ immunostaining results presented diffuse cytoplasmic staining, in accordance with what has been reported by other investigators. ${ }^{3,4,13,22,25,26,30,40}$ Only GH and ACTH were immunohistochemically analyzed in the present study, because we did not include data from patients who presented features suggestive of the oversecretion of other pituitary hormones. Moreover, because we used Pit-1 to identify lesions from acromegalic patients, we did not further differentiate the other lineages that were Pit-1 immunopositive (somatotrophs, lactotrophs, mammosomatotrophs, and thyrotrophs). ${ }^{25}$ Thus, Pit-1-immunopositive 


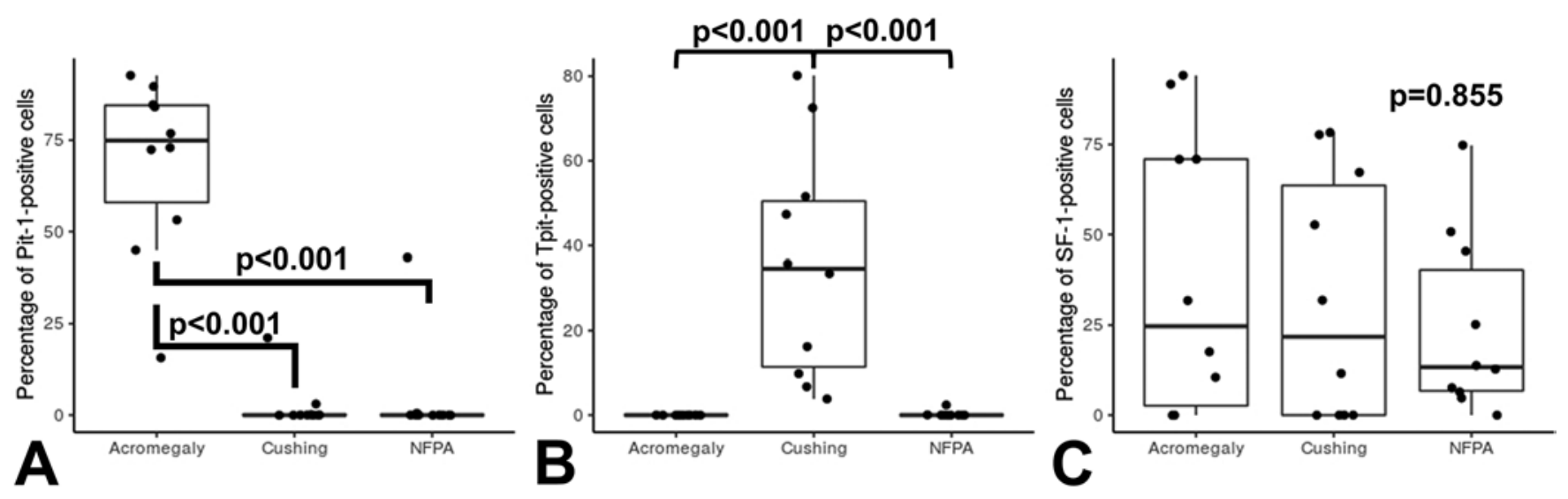

FIG. 3. Analyses of Pit-1, Tpit, and SF-1 expression (\% positive cells/HPF) in PAs per group. A and B: Significantly higher frequencies of Pit-1- and Tpit-positive cells in PAs from acromegalic and Cushing disease patients, respectively. C: Similar SF-1 expression in all three groups (acromegaly, Cushing, NFPA). Details on frequency of positive cells in each PA group are given in the text.

cells in the acromegalic patients were considered to be somatotrophs. Since Tpit has been reported to be indicative of cellular corticotroph differentiation, ${ }^{25}$ we did not perform additional immunohistochemical characterization to identify corticotroph lesions.

Pit-1 and Tpit enabled the identification of lesions associated with acromegaly and Cushing disease, respectively. Such observations are in accordance with previous findings, as Pit-1 may only be detected in specific pituitary cell types, such as somatotrophs. ${ }^{3}$ Similar observations were noticed for corticotroph cells and Tpit, as this factor plays a role in the transcription of proopiomelanocortin (POMC), an ACTH precursor. ${ }^{18,41}$ Therefore, in this study,

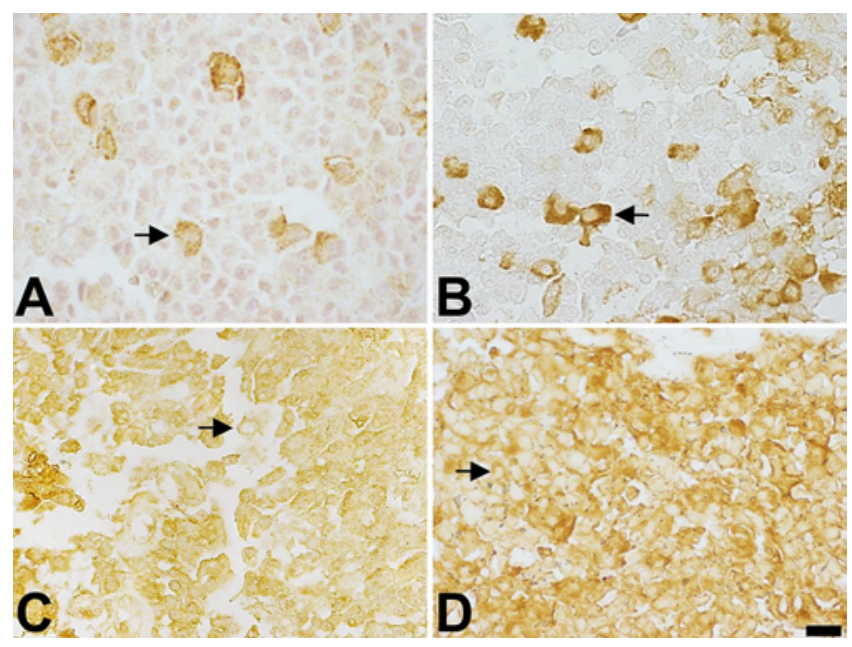

FIG. 4. Comparison between immunostaining patterns for 2 antibodies against growth hormone, $\mathrm{AbGH}-1(\mathbf{A})$ and $\mathrm{AbGH}-2(\mathbf{B})$, in a patient with acromegaly, as well as 2 antibodies against adrenocorticotropic hormone, AbACTH-1 (C) and AbACTH-2 (D), in a patient with Cushing disease. Notice the diffuse cytoplasmic staining pattern irrespective of the antibody used (arrows). Less tissue expression (Mean value) for $\mathrm{GH}$ was detected with $\mathrm{AbGH}-1$ in comparison with $\mathrm{AbGH}-2$, whereas similar levels of ACTH were detected with AbACTH-1 and AbACTH-2. Bar: 30 $\mu \mathrm{m}(\mathrm{A}-\mathrm{D})$.
Pit-1 and Tpit enabled us to differentiate somatotroph and corticotroph lesions, respectively, from NFPAs.

The frequency of SF-1-immunopositive cells was similar in all groups. SF-1 is a transcription factor related to gonadotroph differentiation. ${ }^{4}$ Moreover, the majority of NFPAs are gonadotrophs. ${ }^{25}$ Thus, it would be reasonable to expect that NFPAs presented a higher ratio of SF-1staining cells than somatotrophs and corticotrophs. Indeed, other authors have observed that gonadotrophs are frequently positive for a factor. However, SF-1 expression has also been described in somatotrophs and corticotrophs. ${ }^{4}$ Furthermore, $30 \%$ of the PAs studied in 1 series coexpressed mRNA for Pit-1 and SF-1. ${ }^{23}$ Hence, our finding that SF-1-positive cells may be detected in lesions together with Pit-1 or Tpit corroborates the possibility of coexpression of different transcription factors. Accordingly, our study results indicate that SF-1 alone is not effective in differentiating PAs associated with Cushing or acromegaly from NFPAs.

We evaluated hormonal tissue expression by using ImageJ and 2 different antibodies against GH and ACTH each. Such an approach is to our knowledge original for human specimens, but a similar method employing a digital image analyzer has been reported to evaluate $\mathrm{GH}$ and PRL immunopositivity in rat PAs. The authors of that report, who also evaluated the number of positive cells manually, concluded that the results obtained from digital analyses correlated better with tissue hormonal concentrations assessed by radioimmunoassay. ${ }^{22}$ Such a conclusion supports the digital analysis adopted for the present study. Moreover, we performed immunohistochemical analyses with different antibodies to avoid sensitivity limitations that could hamper epitope detection and/or identification of correlations between PA hormonal expression and laboratory data.

Concerning GH tissue expression, when AbGH-1 was used, there was no statistically significant difference between results for patients with acromegaly and those with NFPA. However, when AbGH-2 was used, tissue expression was higher in patients with acromegaly than individuals with NFPA. Correlations were not observed between 

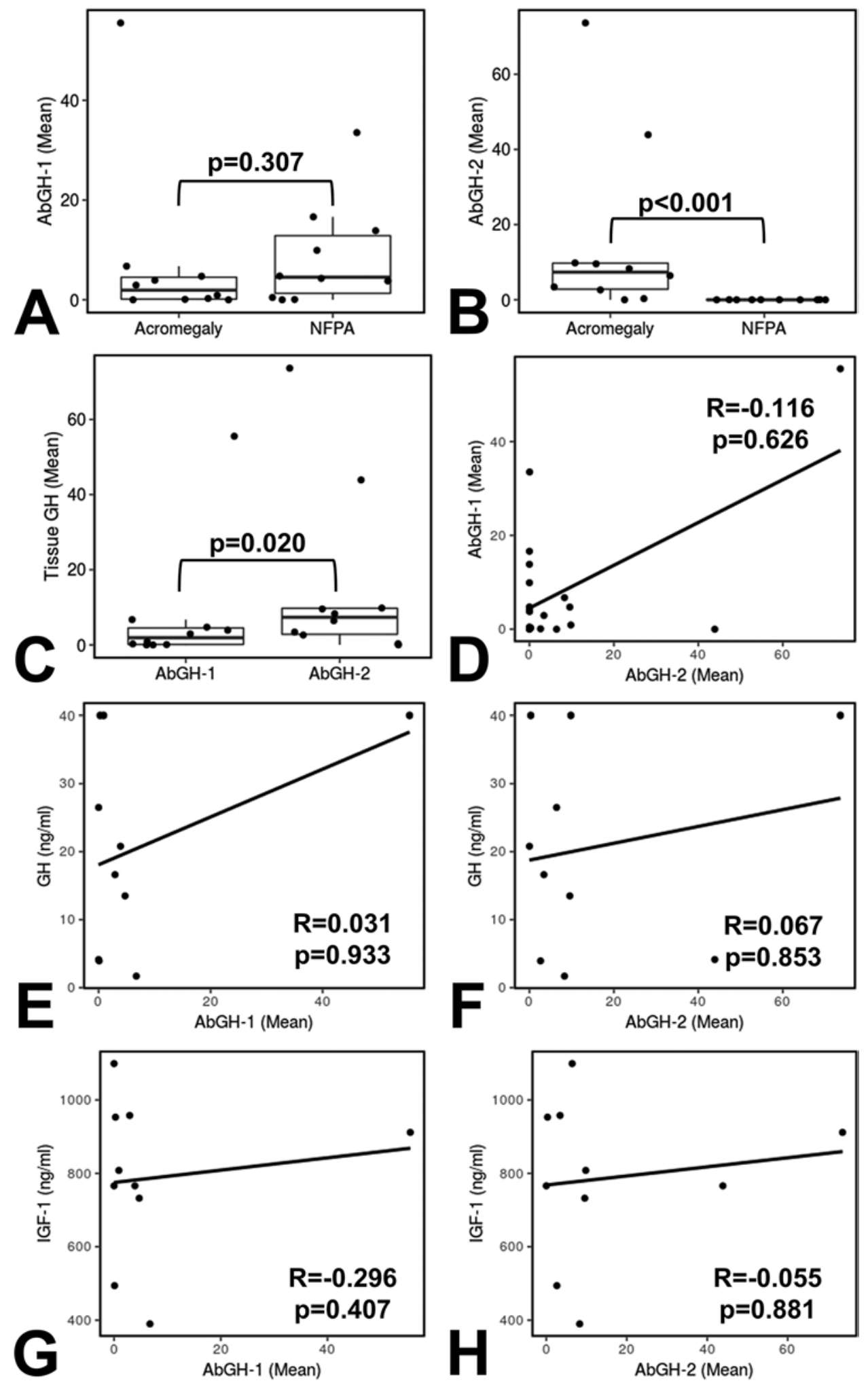

FIG. 5. Analyses of GH expression in PAs by using the ImageJ software and correlations with GH and IGF-1 serum levels. A and B: Tissue expression (Mean value) verified with AbGH-1 (A) or AbGH-2 (B) in the groups of patients with acromegaly or NFPA. Significant differences in $\mathrm{GH}$ expression between the two groups were detected only for AbGH-2. C: Tissue expression of GH (Mean value) in acromegalic individuals detected with AbGH-1 or AbGH-2. Significantly higher Mean values were observed with AbGH-2. D: Correlation of GH tissue expression (Mean value) after using AbGH-1 or AbGH-2 in acromegaly and NFPA patients considered as one group. No correlation was found for the Mean values verified with both antibodies. $\mathrm{E}$ and $\mathrm{F}$ : Correlations between $\mathrm{GH}$ tissue expression (Mean value) obtained with $\mathrm{AbGH}-1$ ( $\mathrm{E}$ ) or AbGH-2 (F) and $\mathrm{GH}$ serum levels (ng/ml). G and H: Correlations between $\mathrm{GH}$ tissue expression (Mean values) observed with $\mathrm{AbGH}-1$ (G) or AbGH-2 (H) and serum IGF-1 (ng/ml). No correlations were found considering both antibodies and the serum levels of the studied molecules. 

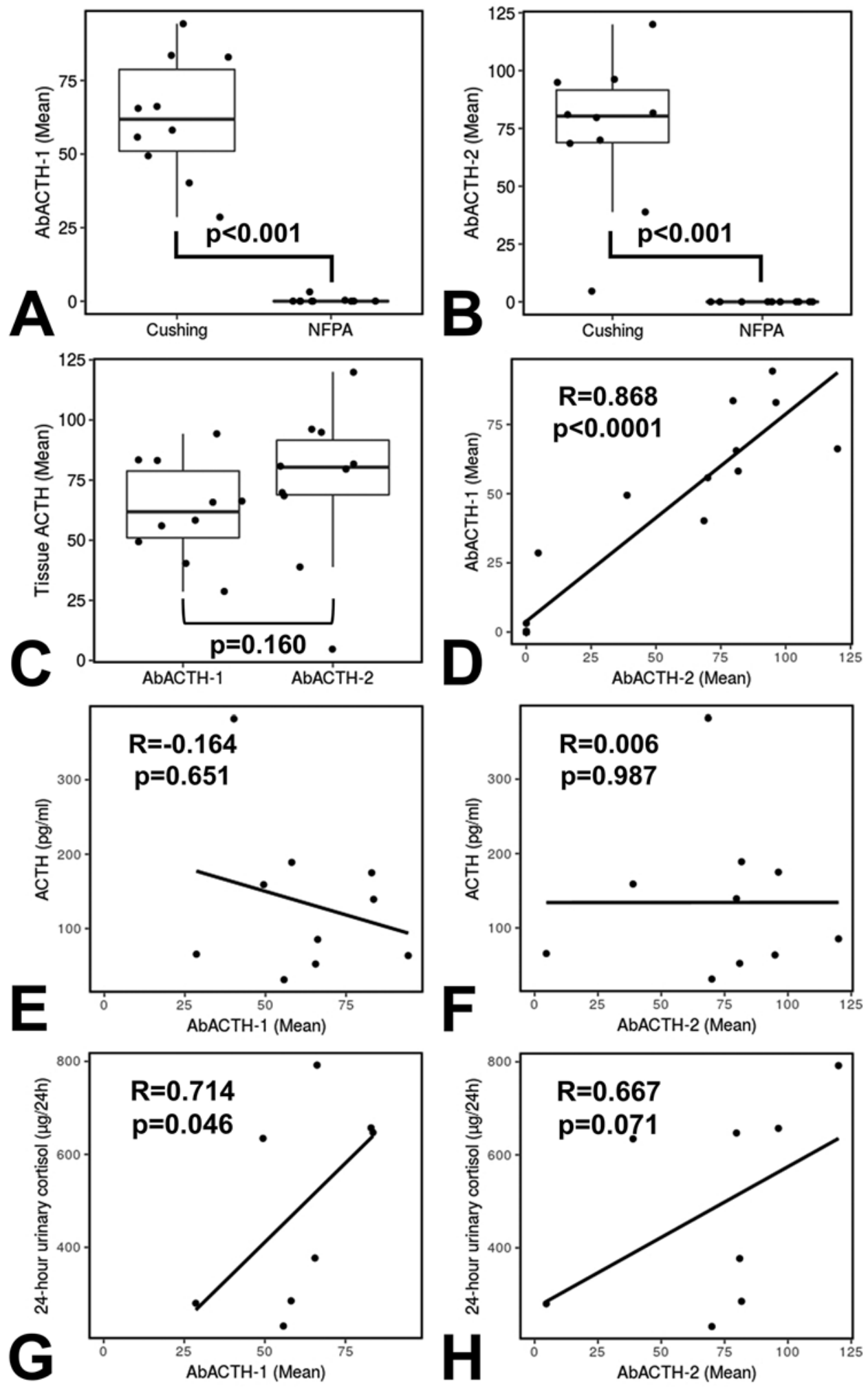

FIG. 6. Analyses of ACTH expression in PA specimens using the Image J software and correlations with ACTH and 24-hour urinary cortisol levels. A and B: Tissue expression (Mean value) evaluated with AbACTH-1 (A) or AbACTH-2 (B) in the Cushing or NFPA groups. A significant difference in Mean values was detected with both antibodies. C: ACTH tissue expression (Mean value) in Cushing disease patients detected with AbACTH-1 or AbACTH-2. No difference was identified. D: Correlation of ACTH tissue expression (Mean value) after using AbACTH-1 or $\mathrm{AbACTH}-2$ in the Cushing disease and NFPA specimens considered as one group. Correlation was detected between Mean values obtained with both antibodies. $E$ and F: Correlations between ACTH tissue expression (Mean value) obtained with AbACTH-1 (E) or AbACTH-2 (F) and ACTH serum levels (pg/ml). G and H: Correlations between ACTH tissue expression (Mean values) assessed by AbACTH-1 (G) or AbACTH-2 $(\mathrm{H})$ and 24 -hour urinary cortisol ( $\mu \mathrm{g} / 24 \mathrm{hrs})$. A correlation was found only between AbACTH-1 and urinary levels. 
the values obtained using each antibody when the acromegaly and NFPA groups were considered together. Furthermore, in acromegalic patients the 2 antibodies each yielded different tissue expressions. Antibody properties may have contributed to such differences, as sensitivity and specificity might differ between antibodies against the same antigen. $8,10,21$ Therefore, our data support the finding that different antibodies against $\mathrm{GH}$ in human PAs may lead to distinct levels of tissue expression assessed by immunohistochemistry.

Regarding PAs associated with acromegaly, our results partially corroborate those of other investigators. In fact, $\mathrm{GH}$ gene expression was increased in PAs that were immunopositive for GH compared to null PAs (without immunostaining for adenopituitary hormones). ${ }^{42}$ Similarly, it has been demonstrated that $\mathrm{GH}$-secreting PAs overexpressed the GH gene compared to normal pituitary tissue, whereas NFPAs underexpressed the same gene. ${ }^{28}$ Those findings are compatible with data obtained using AbGH2 , but at variance with the results obtained with AbGH-1. Therefore, it is possible that detection of increased immunostaining for $\mathrm{GH}$ in acromegalic patients depends on antibody selection.

For ACTH tissue expression, values were higher in PAs from patients with Cushing disease than in lesions from patients with NFPA, irrespective of the antibody. Moreover, a significant correlation was found between ACTH expression obtained with each antibody when the Cushing and NFPA groups were analyzed together. Also, both antibodies detected similar ACTH expression in PAs from Cushing patients. Therefore, our data favor similar sensitivity and specificity between both antibodies. Previous studies support our observations regarding ACTH expression in PAs associated with Cushing disease. It was found that gene expression of POMC was higher in ACTH-immunopositive PAs than null lesions. ${ }^{42}$ Moreover, increased or diminished POMC gene expression in PAs that were marked for ACTH or NFPA, respectively, has been described.$^{28}$ Therefore, our data corroborate increased ACTH levels in PAs from Cushing patients. Taken together, our results regarding $\mathrm{ACTH}$ and $\mathrm{GH}$ tissue expression in PAs suggest that it would be necessary to use antibodies with sufficient sensitivity and specificity to recognize levels of tissue expression that would be compatible with clinical features of hormone excess.

We did not find correlations between GH or ACTH serum levels and their respective tissue expressions, regardless of antibody. These results might be due to the fact that immunostaining for a hormone may reflect a transitory state of intracellular storage and not secretory activity. ${ }^{12-15,37}$ Similarly, a correlation between GH and PRL immunostaining and tissue concentrations of those hormones assessed by radioimmunoassay was reported in rat PAs. ${ }^{22}$ Therefore, immunohistochemical analysis does not evaluate PA endocrine activity at a given time, but rather tissue storage, which could account for the fact that we found no correlation between lesion expression and peripheral levels of hormones. Indeed, a relation between immunostaining for and serum levels of GH was reported, whereas other authors did not observe such a correlation. ${ }^{12,13,30}$ As for ACTH, no correlation between PA immunostaining and corresponding serum levels was found. ${ }^{12}$ Therefore, the lack of correlation between tissue immunostaining and peripheral levels of ACTH and GH may be due to the inadequacy of immunohistochemical analysis to evaluate momentary endocrine activity.

We also tested for correlations between GH and ACTH tissue expression and concentrations of functionally associated molecules. Immunostaining for the first hormone did not correlate with IGF-1 concentrations regardless of the antibody used. It has been reported that in acromegalic individuals, linear relationships between IGF-1 and $\mathrm{GH}$ levels occur only for low concentrations of the second molecule. Moreover, acromegalic patients with similar $\mathrm{GH}$ values may present a wide range of IGF-1 levels. ${ }^{17,31}$ Thus, since there is no correlation between tissue expression and peripheral concentration of $\mathrm{GH}$, as well as between $\mathrm{GH}$ and IGF-1 levels, it would be reasonable that $\mathrm{GH}$ tissue expression would not correlate with IGF-1 blood concentrations.

We verified a correlation between ACTH immunostaining and 24-hour urinary cortisol only with AbACTH-1. Specifically, the total amount of cortisol eliminated in urine during 24 hours would be less subjected to variations than cortisol serum levels. Indeed, it is known that both cortisol and ACTH serum levels present variations during the circadian cycle, which may have further variations in Cushing patients. 6,7,16,39 Therefore, detection of urinary cortisol levels would be an approach to compensate for unpredictable variations of ACTH/cortisol, increasing the chances of finding correlations with ACTH tissue expression. Finally, the fact that we were able to detect correlations between PA ACTH and 24-hour urinary cortisol levels with only 1 antibody may be explained by specificity and sensitivity differences between the antibodies.

\section{Study Limitations}

Our investigation was limited by the use of only 2 antibodies to detect hormone tissue expression and by the focus on hormones related to some of the pituitary lesions for which treatment can be approached surgically, that is, $\mathrm{GH}$ and ACTH. The use of only 2 antibodies raises the question of how other similar molecules would perform. Considering the present results, it is not possible to estimate whether other $\mathrm{GH}$ antibodies would present more accurate correlations with clinical laboratory data. The opposite is valid for ACTH. Therefore, even though it was not our intention to assess the performance of specific commercial reagents, our study illustrates that different antibodies might lead to different immunohistochemical results, and thus the present results may not necessarily be extrapolated to PAs associated with other clinical manifestations.

\section{Conclusions}

Our results show that differences in technical features of antibodies used against the same antigen may lead to the detection of different hormonal immunopositivity results in a PA specimen assessed by digital analysis. Moreover, we found no correlation between hormonal immunostaining and blood concentrations of GH, IGF-1, and ACTH. 
However, ACTH expression may correlate with 24-hour urinary cortisol depending on antibody properties. Also, the use of transcription factors, as recommended by the 2017 WHO classification, accurately identified lesions associated with acromegaly and Cushing disease, as shown by the relation verified between transcription factor immunostaining and presurgical clinical manifestations. These findings highlight the relevance of transcription factors to PA classification because, even with a digital tool, it was not possible to verify a reliable correlation between clinical laboratory data and tissue hormonal immunopositivity.

\section{Acknowledgments}

The authors thank Mr. Helymar C. Machado for helping with statistical analysis.

\section{References}

1. Abdelmannan D, Chaiban J, Selman WR, Arafah BM: Recurrences of ACTH-secreting adenomas after pituitary adenomectomy can be accurately predicted by perioperative measurements of plasma ACTH levels. J Clin Endocrinol Metab 98:1458-1465, 2013

2. Agustsson TT, Baldvinsdottir T, Jonasson JG, Olafsdottir E, Steinthorsdottir V, Sigurdsson G, et al: The epidemiology of pituitary adenomas in Iceland, 1955-2012: a nationwide population-based study. Eur J Endocrinol 173:655-664, 2015

3. Asa SL, Bamberger AM, Cao B, Wong M, Parker KL, Ezzat $\mathrm{S}$ : The transcription activator steroidogenic factor-1 is preferentially expressed in the human pituitary gonadotroph. J Clin Endocrinol Metab 81:2165-2170, 1996

4. Asa SL, Puy LA, Lew AM, Sundmark VC, Elsholtz HP: Cell type-specific expression of the pituitary transcription activator pit-1 in the human pituitary and pituitary adenomas. $\mathbf{J}$ Clin Endocrinol Metab 77:1275-1280, 1993

5. Bassetti M, Arosio M, Spada A, Brina M, Bazzoni N, Faglia G, et al: Growth hormone and prolactin secretion in acromegaly: correlations between hormonal dynamics and immunocytochemical findings. J Clin Endocrinol Metab 67:1195-1204, 1988

6. Berson SA, Yalow RS: Radioimmunoassay of ACTH in plasma. J Clin Invest 47:2725-2751, 1968

7. Bierwolf C, Kern W, Mölle M, Born J, Fehm HL: Rhythms of pituitary-adrenal activity during sleep in patients with Cushing's disease. Exp Clin Endocrinol Diabetes 108:470-479, 2000

8. Borrisholt M, Nielsen S, Vyberg M: Demonstration of CDX2 is highly antibody dependent. Appl Immunohistochem Mol Morphol 21:64-72, 2013

9. Daly AF, Rixhon M, Adam C, Dempegioti A, Tichomirowa MA, Beckers A: High prevalence of pituitary adenomas: a cross-sectional study in the province of Liege, Belgium. J Clin Endocrinol Metab 91:4769-4775, 2006

10. Gown AM: Diagnostic immunohistochemistry: what can go wrong and how to prevent it. Arch Pathol Lab Med 140:893-898, 2016

11. Gruppetta M, Mercieca C, Vassallo J: Prevalence and incidence of pituitary adenomas: a population based study in Malta. Pituitary 16:545-553, 2013

12. Herzog T, Schlote W, Lorenz R, Jungmann E, Althoff PH: Pituitary adenomas: serum hormone levels and immunohistochemical staining for ACTH, GH and prolactin. Clin Neuropathol 12:117-120, 1993

13. Irsy G, Góth M, Bálint K, Slowik F, Szabolcs I, Pásztor E, et al: Comparison of the serum hormone levels and histological findings in pituitary adenomas. Exp Clin Endocrinol 88:224-236, 1986
14. Kameya T, Tsumuraya M, Adachi I, Abe K, Ichikizaki K, Toya S, et al: Ultrastructure, immunohistochemistry and hormone release of pituitary adenomas in relation to prolactin production. Virchows Arch A Pathol Anat Histol 387:31-46, 1980

15. Kovacs K, Horvath E, Corenblum B, Sirek AM, Penz G, Ezrin C: Pituitary chromophobe adenomas consisting of prolactin cells: a histologic, immunocytological and electron microscopic study. Virchows Arch A Pathol Anat Histol 366:113-123, 1975

16. Krieger DT, Allen W, Rizzo F, Krieger HP: Characterization of the normal temporal pattern of plasma corticosteroid levels. J Clin Endocrinol Metab 32:266-284, 1971

17. Lamberts SWJ, Uitterlinden P, Verleun T: Relationship between growth hormone and somatomedin- $\mathrm{C}$ levels in untreated acromegaly, after surgery and radiotherapy and during medical therapy with sandostatin (SMS 201-995). Eur J Clin Invest 17:354-359, 1987

18. Lamolet B, Pulichino AM, Lamonerie T, Gauthier Y, Brue $\mathrm{T}$, Enjalbert A, et al: A pituitary cell-restricted $\mathrm{T}$ box factor, Tpit, activates POMC transcription in cooperation with Pitx homeoproteins. Cell 104:849-859, 2001

19. Lloyd RV, Cano M, Chandler WF, Barkan AL, Horvath E, Kovacs K: Human growth hormone and prolactin secreting pituitary adenomas analyzed by in situ hybridization. Am J Pathol 134:605-613, 1989

20. Lloyd RV, Osamura YR, Kloppel G, Rosai J: WHO Classification of Tumours Of Endocrine Organs, ed 4. Geneva: WHO, 2017

21. Matoso A, Singh K, Jacob R, Greaves WO, Tavares R, Noble $\mathrm{L}$, et al: Comparison of thyroid transcription factor-1 expression by 2 monoclonal antibodies in pulmonary and nonpulmonary primary tumors. Appl Immunohistochem Mol Morphol 18:142-149, 2010

22. Matsuo T, Mori H, Nishimura Y, Maeda T, Nakagawa JI, Obashi A: Quantification of immunohistochemistry using an image analyser: correlation with hormone concentrations in pituitary adenomas. Histochem J 27:989-996, 1995

23. McDermott MT, Haugen BR, Gordon DF, Wood WM, Brown NS, Bauer CA, et al: Reverse transcription polymerase chain reaction analysis of pituitary hormone, Pit-1 and steroidogenic factor-1 messenger RNA expression in pituitary tumors. Pituitary 2:217-224, 1999

24. Mete O, Asa SL: Clinicopathological correlations in pituitary adenomas. Brain Pathol 22:443-453, 2012

25. Mete O, Cintosun A, Pressman I, Asa SL: Epidemiology and biomarker profile of pituitary adenohypophysial tumors. Mod Pathol 31:900-909, 2018

26. Mete O, Lopes MB: Overview of the 2017 WHO Classification of Pituitary Tumors. Endocr Pathol 28:228-243, 2017

27. Molitch ME: Diagnosis and treatment of pituitary adenomas: a review. JAMA 317:516-524, 2017

28. Morris DG, Muşat M, Czirják S, Hanzély Z, Lillington DM, Korbonits M, et al: Differential gene expression in pituitary adenomas by oligonucleotide array analysis. Eur J Endocrinol 153:143-151, 2005

29. Mota MVB, Zaidan BC, do Canto AM, Ghizoni E, Tedeschi H, de Souza Queiroz L, et al: ATP synthase subunit beta immunostaining is reduced in the sclerotic hippocampus of epilepsy patients. Cell Mol Neurobiol 39:149-160, 2019

30. Mukai K: Pituitary adenomas. Immunocytochemical study of 150 tumors with clinicopathologic correlation. Cancer 52:648-653, 1983

31. Oldfield EH, Jane JA Jr, Thorner MO, Pledger CL, Sheehan JP, Vance ML: Correlation between GH and IGF-1 during treatment for acromegaly. J Neurosurg 126:1959-1966, 2017

32. Petersenn S, Newell-Price J, Findling JW, Gu F, Maldonado M, Sen K, et al: High variability in baseline urinary free 
cortisol values in patients with Cushing's disease. Clin Endocrinol (Oxf) 80:261-269, 2014

33. Raappana A, Koivukangas J, Ebeling T, Pirilä T: Incidence of pituitary adenomas in Northern Finland in 1992-2007. J Clin Endocrinol Metab 95:4268-4275, 2010

34. Reinking L. ImageJ basics (Version 1.38). Accessed April 6, 2020. https://imagej.nih.gov/ij/docs/pdfs/ImageJ.pdf

35. Ross RJM, Grossman A, Bouloux P, Rees LH, Doniach I, Besser GM: The relationship between serum prolactin and immunocytochemical staining for prolactin in patients with pituitary macroadenomas. Clin Endocrinol (Oxf) 23:227235, 1985

36. Sandret L, Maison P, Chanson P: Place of cabergoline in acromegaly: a meta-analysis. J Clin Endocrinol Metab 96:1327-1335, 2011

37. Schatz H, Daun M, Leicht R, Stracke H, Saeger W, Zierski J: Immunohistochemical examination of pituitary adenomas. Comparison to clinical and endocrinological findings. Horm Res 21:246-253, 1985

38. Schroeder JL, Spiotta AM, Fleseriu M, Prayson RA, Hamrahian $\mathrm{AH}$, Weil RJ: Absence of immunostaining for growth hormone in a subset of patients with acromegaly. Pituitary 17:103-108, 2014

39. Sederberg-Olsen P, Binder C, Kehlet H, Neville AM, Nielsen LM: Episodic variation in plasma corticosteroids in subjects with Cushing's syndrome of differing etiology. J Clin Endocrinol Metab 36:906-910, 1973

40. Sen A, Das C, Mukhopadhyay M, Mukhopadhyay S, Deb S, Mukhopadhyay B: Cytohistological correlation in pituitary tumor and immunological assessment with the help of Ki-67. J Postgrad Med 63:96-99, 2017

41. Tani Y, Sugiyama T, Izumiyama H, Yoshimoto T, Yamada S, Hirata Y: Differential gene expression profiles of POMCrelated enzymes, transcription factors and receptors between non-pituitary and pituitary ACTH-secreting tumors. Endocr J 58:297-303, 2011
42. Zebracka-Gala J, Rudnik A, Hasse-Lazar K, Larysz D, Jarząb M, Krajewska J, et al: Molecular classification of pituitary adenomas: in search for criteria useful for highthroughput studies. Endokrynol Pol 67:148-156, 2016

\section{Disclosures}

The authors report no conflict of interest concerning the materials or methods used in this study or the findings specified in this paper.

\section{Author Contributions}

Conception and design: Rogerio, Tamanini, Dal Fabbro. Acquisition of data: all authors. Analysis and interpretation of data: Rogerio, Tamanini, Dal Fabbro. Drafting the article: Rogerio, Tamanini, Dal Fabbro. Critically revising the article: Rogerio, Tamanini, Dal Fabbro, Queiroz. Reviewed submitted version of manuscript: Rogerio, Tamanini, Dal Fabbro. Approved the final version of the manuscript on behalf of all authors: Rogerio. Statistical analysis: Rogerio, Tamanini, Dal Fabbro. Administrative/technical/material support: Rogerio, Tamanini, Dal Fabbro. Study supervision: Rogerio, Tamanini, Dal Fabbro.

\section{Correspondence}

Fabio Rogerio: School of Medical Sciences, University of Campinas (UNICAMP), Campinas, SP, Brazil. fabio@fcm.unicamp.br. 